opposite side of the nest, replacing the unscented one there. "Thus there was an unscented path in the position of the old trail and the old familiar scented path was in a new position."

The ants now, if they are 'reflex machines' " should either have spent approximately as much time learning the way down the new incline as they did before, or else, in their random movements they should have happened upon the scented incline and gone down it."

But the ants went almost immediately down the unscented path! These group experiments were controlled and the results there obtained were affirmed by using marked individual ants. By further experiments, Turner shows that the direction of the light plays an important rôle in determining the course of the ants.

Other interesting statements are made in the Note, but the details of the experiments leading to them are omitted until his complete paper appears.

J. B. W.

\title{
The Habits, Instincts and Mental Powers of Spiders, Genera
} Argiope and Epeira. James P. Porter. Am. Jr. of Psychol., I906, XVII., 306-357.

On the historical side of this paper, Dr. Porter has gleaned from various sources and put into summarized form the main psychological facts of interest concerning the behavior of spiders. This part of the work is so well and compactly done that one is almost immediately given the proper setting to the writer's own observations.

To better observe the reactions of the spiders, they were brought into the laboratory and supplied with as natural an environment as was possible. Such subjects as the choice of a place for a web, the nest and material for the nest, the manner of building the web and its variability are discussed. A quantitative measure of the variability of instinct was determined by counting the elements in parts of the webs.

Further instinctive activities were studied, such as the time of spinning the web, and the stimulus which releases the movements concerned in this act, feeding habits, web-shaking, mating, etc.

Some controlled experimental work was done towards testing the factor of adaptability in the reactions of these spiders, but the present work is merely 'eine bahnbrechende Untersuchung'-Dr. Porter promising a later experimental paper.

This type of study will have to be undertaken more and more often now, as comparative psychologists begin the study of new forms. The older studies by the general 'naturalist' are practically worthless as a point of departure for scientific work on behavior.

T. B. W. 